\title{
THERMAL INDUCED CONDUCTANCE OF KOH ETCHED SILICON SURFACE
}

\author{
Shobha Kanta Lamichhane \\ Tribhuvan University, Prithwi Narayan Campus, Pokhara (Nepal) \\ Corresponding author: sklamichhane@ hotmail.com \\ Received 23 June, 2009; Revised 3 March, 2010
}

\begin{abstract}
Anisotropic $\mathrm{KOH}$ etch rate of silicon is found to vary with thermal agitation along with crystal plane orientation. In this work, experimental results of etch rate is found with their unusual values of activation energy along different planes. The various sites that an atom can occupy are not equivalent of their energy; some are more favorable to removal than others. In this paper attention is given to demonstrate thermal activation influences the behavior of etching mechanism as well as surface morphology. Low-voltage contact mode atomic force microscopy (AFM) has been employed to analyze the morphology of the etched silicon surface at relevant temperature. With temperature evolution the width of the forbidden energy gap is going down and hence conductivity is rises.
\end{abstract}

Keywords: Anisotropy, Etch rate, MEMS, SOI, AFM, Contact mode.

\section{INTRODUCTION}

Micro-electro mechanical system (MEMS) devices are typically made from silicon (Kovacs, 1998) using conventional semiconductor manufacturing techniques. They can be either etched from larger structure or built up by employing material deposition processes (Finne and Klein, 1967). Silicon micromachining technology has become popular for the fabrication of a number of MEMS devices (Perterson, 2001). Silicon micromachining provides proper dimensional control and consistent mechanical properties and is now established as a mature technology for micro devices. It allows us to control precisely the shape and dimension of microstructure (Abott et al., 1993). The technology is now being used to fabricate sensors for measuring pressure, force, flow and acceleration using the advantage of batch processing.

Etching silicon strongly depends on orientation of crystal plane and hence anisotropy. Various experimental techniques have been developed to study anisotropic etching of silicon. Etching silicon can perform with various etchants, such as KOH, TMAH and EDP (Campbell et al., 1995) are few examples. The dissolution of silicon is needed for deep etching and micromachining, shaping and cleaning. Taking out silicon atoms from its surface with time for a given temperature and concentration of $\mathrm{KOH}$ is not same for different plane orientations. Its flexibility is exploited to design microstructures and devices with fraction of micron resolution (Schwartz and Robbin, 1961) nowadays. 
For a given temperature and concentration, removal of silicon atoms is not same for all silicon crystal planes. The moving surface with time depends on etchant's temperature (Campbell et al., 1995) and etchant's concentration (Abott et al., 1993) as well as doping of silicon substrate (Lee, 1969). A high etching rate is generally desirable in a manufacturing environment. Too high etch rate however may render a process difficult to control because during etching, hydrogen bubbles (Finne and Klein, 1967) are formed on silicon surface. Thus, pyramid formations (Abott et al., 1993) can be related to poor bubble detachment, precluding diffusion to specific point on the surface. However, anisotropic etching has provided as a tool for shaping of silicon structures and as an evaluation of protective surface films on silicon substrate (Schwartz and Robbin, 1961). Due to diverse functional dependence of etch rate (ER), a complete study of this system is extremely complicated. So, accuracy of ER may vary from laboratory to laboratory and accuracy level of measurement techniques. In this study, ER of silicon and its morphological microstructure as observed by AFM has been discussed.

The operation of scanning tunneling microscope (STM) is direct consequence of electrons in quantum mechanical tunneling through a potential barrier. In practice, metal tip is brought (by piezo-electric device) very close (angstrom order) to the surface of the sample to be studied. When the bias voltage is applied to the tip or sample, electrons are attracted by whatever side holds positive potential. The gap between the tip and sample becomes a finite barrier potential for electrons that are nearly free, and as per quantum theory, electrons begin to tunnel through the barrier. The tip is then rastered across the two dimensional surface of the sample and current is recorded. The current is use for information about the topography and electronic structure of the sample surface. Measurement of various inter-related tunneling parameters, e.g., bias voltage, tunneling current, tunneling gap etc one can knows varieties of surface electronic property of the sample.

STM is based on quantum tunneling between two electrodes separated by a fixed distance. Suppose the electrodes are taken as the tip and the sample such that their separation is ' $d$ ' with work functions ' $\phi_{\mathrm{t}}$ ' and ' $\phi_{\mathrm{s}}$ '. When bias voltage between tip and sample is small and positive, $\mathrm{V}_{\text {bias }} \ll\left(\phi_{\mathrm{t}}+\phi_{\mathrm{s}}\right) / 2$, the barrier between the two electrodes can approximated as a rectangular potential whose current is determined by solving the Schordinger wave equation.

\section{MATERIALS AND METHODS}

Silicon wafer of thermally bonded silicon on insulator (SOI) with (100) orientation was used in this study. The thickness of the top silicon buried $\mathrm{SiO}_{2}$ layer and bottom silicon were 2 and $450 \mathrm{~nm}$ respectively. The wafers were of p-type silicon with a resistivity of $4-$ $631022 \mathrm{~V}-\mathrm{cm}$ for the top silicon and 12-15 V-cm for the bottom silicon. Only the top silicon layer was etched by the KOH solution. First, the SOI wafers were prepared with standard RCA cleaning which consist of two consecutive cleaning solutions (standard clean1, i.e. SC1 clean). Radio Corporation of America, Standard Clean 1 (RCASC1) Cleaning including $\mathrm{H}_{2} \mathrm{O}-\mathrm{H}_{2} \mathrm{O}_{2}-\mathrm{NH}_{4} \mathrm{OH}$ with volume ratio typically 5:1:1 was adopted in many steps during experimentation since it removes organic contaminants and particles 
physically attached to a wafer surface. A $450 \AA$ layer of $\mathrm{Si}_{3} \mathrm{~N}_{4}$, which acts as a $\mathrm{KOH}$ etching mask, was deposited on each wafer using low pressure chemical vapor deposition (LPCVD). Oxide can be used as an etch mask for short period in the $\mathrm{KOH}$ solution, but for long periods, nitride is a better etch mask as it etches more slowly. The SOI wafer was then cut into $10 \mathrm{~mm} \times 10 \mathrm{~mm}$ pieces. With the dice pieces held on a vacuum chuck, positive photoresist, OCG 825 , was used to pattern the $\mathrm{Si}_{3} \mathrm{~N}_{4}$ for the $\mathrm{KOH}$ etching mask. The nitride was etched with CF4 plasma and then the exposed portion of the top silicon on each SOI wafer was etched in $\mathrm{KOH}$ solution of varying temperature and concentration. To assure the sample free of particulate and other airborne contaminants, the experiment were conducted in a dust free room.

Samples prepared in this way, were ready for measurements and microscopic observations by AFM. Images were taken in low voltage contact mode. Before performing AFM measurement, it was ascertained with the help of spirit level and AFM software, scanner plane and AFM head plane were made parallel. During each measurement $0.1 \mu \mathrm{m}$ was taken as scan size. Finally, images were recorded in contact mode AFM. Etch rate, surface roughness (curve for height vs. topographical distance), activation energy of etched surfaces is chosen as the parameter for analysis.

The STM of Thermo-microscope, USA, Auto probe Electronic Module; Model no 1000 by which we took these readings. Scanning was done in the constant current mode at a positive sample bias of $100 \mathrm{mV}$. Composition of the tip was $80 \% \mathrm{Pt}$ and $20 \% \mathrm{Ir}$. The tunneling spectra were taken in ambient condition at various points and collected while STM showed consistent and reproducible curves. To measure tunneling spectra, the sample bias was ramped from $+4 \mathrm{~V}$ to $-2 \mathrm{~V}$ with respect to the tip and tunneling current was monitored. The voltage axis in the tunneling spectrum represents the potential applied to the sample relative to the tip.

\section{RESULTS AND DISCUSSION}

2D moving surface is taken at an ambient condition for understanding etching of silicon. Etch rate is one of the prominent parameter of it whose variation along primary crystallographic orientation as they etched in $\mathrm{KOH}$ environment with temperature are found to vary. Results are displayed in Fig.1 which tells about ER is not a linear function with temperature. ER along three primary planes viz. (111), (110) and (100) with temperature, which indicate (111) plane etches slow compared to other two planes: (100) and (110). 


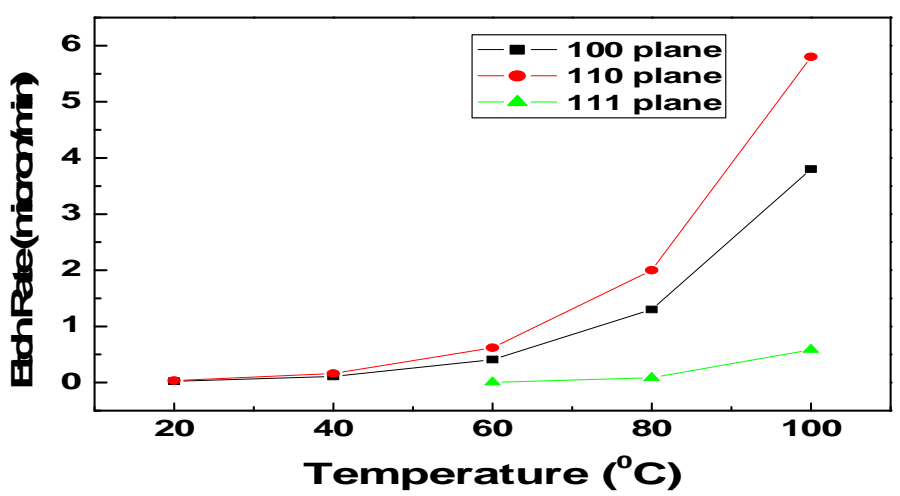

Fig 1: anisotropic etching of silicon

It is obvious from Fig. 1 that ER is a function of temperature. In (111) plane ER rises little sharper beyond $80^{\circ} \mathrm{C}$ where as (110) and (100) plane is stipend before $80^{\circ} \mathrm{C}$. Based on these results, properties of ER in silicon are highly anisotropic. Hence, single crystal silicon has different mechanical characteristics (Perterson, 2001) with respect to orientation. In order to understand basic mechanism of anisotropy in it, one should understand activation energy. Activation energy is the amount of energy need to start a chemical reaction. The rate of reaction depends on the temperature at which it runs. ER and surface morphology are macroscopic results (Gosalvez and Nieminen, 2003). As the moving surface reaches a steady state it seem to fixes etch rate. As a result, final shape of the etched wafer depends on relative etching speed along the crystallographic planes.

Etch rate (ER) depends on average of the microscopic activation energy and existence of fluctuation in fraction of particles at a fixed temperature i.e. on $p_{i}$ and $T$ (Wenspoek and Jansen, 1998) as:

$$
E R=\frac{\Delta Z_{c . m .}}{\Delta t}=\frac{1}{\Delta t} \sum_{i}\left(\Delta Z_{c . m .}\right)_{i .} p_{i}
$$

Where $\Delta Z_{c . m}$ is distance traveled by the center of mass of the surface, $p i$ - is the removal probability of the surface atom $i$, is given by $p_{i}=p_{0 i} e^{-E_{i} / k_{B} T}$, where $T$ is temperature, $k_{B}$ is Boltzmann constant, $E_{i}$ is microscopic activation energy for removal of site $i$ and $p_{o i}$ is a prefactor. So as to understand role of temperature in microscopic energy evolution one should understand reaction mechanism takes place during etching. The chemical mechanism behind it is removal of silicon atom in $\mathrm{KOH}$ solution takes place in two steps (Roher Gregory, 2001). First, four electrons are affected in bulk silicon

$$
\mathrm{Si}+4(\mathrm{OH})^{-} \rightarrow \mathrm{Si}(\mathrm{OH})_{4}+4 e^{-}
$$

In second step, the electrons are released back into the solution accordingly

$$
4 e^{-}+4 \mathrm{H}_{2} \mathrm{O} \rightarrow 4(\mathrm{OH})^{-}+2 \mathrm{H}_{2}
$$

Products in first step $\mathrm{Si}(\mathrm{OH})_{4}$, is supposed to soluble in water. But actually, $\mathrm{Si}(\mathrm{OH})_{4}$ is decompose into water and silicon-dioxide, as a result of supplied thermal energy and 
hence there is removal of silicon atom with release of oxygen gas. The probability of removal of particular silicon atom depends on temperature and microscopic activation energy $\left(E_{i}\right)$ as mentioned. Thus, quantitative estimation of microscopic activation energy will answer these queries.

The quantitative characterization of the activation energy with temperature follows an Arrhenius nature (Gosalvez and Nieminen, 2003) is displaced in Fig. 2. This choice is mathematically guaranteed macroscopic observation, ER, is the result of combination of microscopic activation energies of all atoms (Wang et al., 1999).

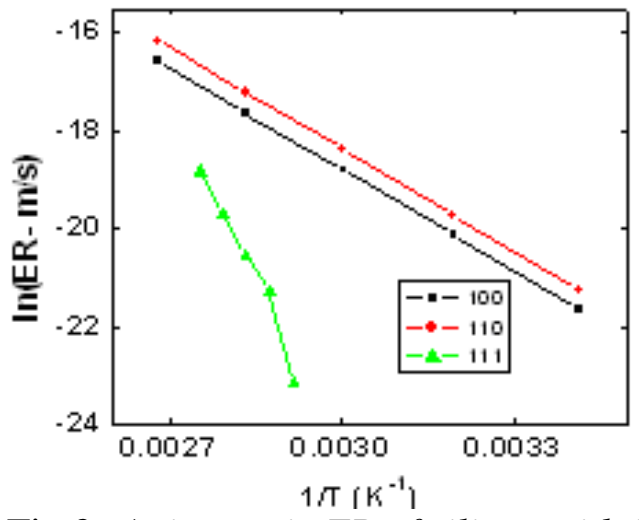

Fig 2: Anisotropic ER of silicon with its Arrhenius plot

Based on slope of Arrhenius plot, $E_{i}$, is now determined: for (111): $0.79 \mathrm{eV}$, for (110): $0.53 \mathrm{eV}$, for (100): $0.55 \mathrm{eV}$ are in the line as reported in the literature (Wang et al. 1999). These quantitative data justify that why ER is slow in (111) and very fast in (110), i.e., energy needed to remove a single atom is entirely different along the plane taken. Hence the plane which has higher activation energy has slow ER (Hennry Wise and Jacques Oudar, 1990) and vice versa. This is because (111) is closely packed; only few atoms have enough energy to cross the barrier to reach metastable state. Note that energy required to displacing an atom from its site i.e., breakdown of the bond and move to an energy crest before descending into a metastable position is associated with activation energy.

Normal silicon has covalent bond structure. If it is in excited state, activation energy must overcome to associated covalent energy between silicon molecules. During etching, activation energy should be speed up for removal of the molecule. Cohesive energy $\left(E_{C}\right)$ of a solid is the energy required to disassemble into constituent parts (Cottam et al., 1989). If we go down to atomic structure, cohesive energy is basically based on LCAO (Linear Combination of Atomic Orbital) model (Roher Gregory, 2001). According to this model when a crystal is formed from independent atoms, number of orbital remains same and retains much of their atomic character. However, there are continuous changes in the energies of the orbital with atomic separation. As the atoms are brought closer together, the orbits begin to interact and hence their bond strength modifies which determines ultimately the physical properties of the crystal. 
Each silicon atom has four valence electrons, which can form strong s- $\mathrm{p}^{3}$ hybrid as in diamond structure (Mayer, 1998) so that energy cost for forming hybrid is s- $\mathrm{p}^{3}$ splitting. As a result, the interaction between orbital should follow inverse square law. If we consider inter-atomic separation $(d)$ as interplanar distance then covalent energy must be activation energy. For known interplanar separation, activation energy along the crystallographic planes is now computed with the help of the equation, $E_{a}=-\frac{4.37 \hbar^{2}}{m d^{2}}$ whose values as obtained are:

\begin{tabular}{lll}
\multicolumn{3}{c}{ Data for activation energy } \\
\hline \hline $\begin{array}{l}\text { Crystal } \\
\text { plane }\end{array}$ & $\begin{array}{l}\text { Interplanar } \\
\text { distance }\left({ }^{0} \mathrm{~A}\right)\end{array}$ & $\begin{array}{l}\text { Cohesive } \\
\text { energy }(\mathrm{eV})\end{array}$ \\
\hline & & \\
$(100)$ & 5.43 & 1.13 \\
$(110)$ & 3.84 & 2.25 \\
$(111)$ & 2.35 & 6.02 \\
\hline \hline
\end{tabular}

Cohesive energy is a function of nearest neighbor distance (Van Vlack, 1970). Thus, greater the cohesive energy more will be activation energy. We now arrive into the conclusion that cohesive energy measures the strength of the interatomic forces and hence the stability of the crystallographic planes. This also support why ER is less along (111).

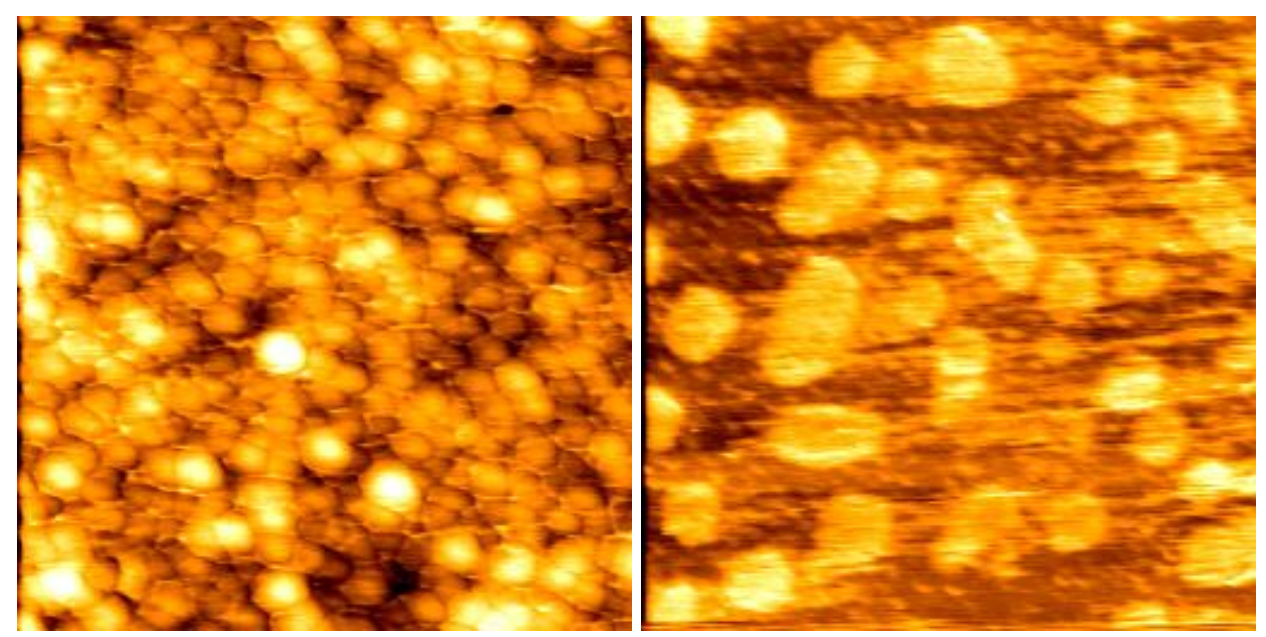

Fig 3: AFM micrograph of etched silicon at $75^{\circ} \mathrm{C}$ and at $95^{\circ} \mathrm{C}$ (scan size $2 \mu \mathrm{m} \times 2 \mu \mathrm{m}$ each)

Above absolute zero there is gradual increase in the amplitude of atomic vibrations. As the temperature of the system increases, the number of molecules that carryout enough energy to react also increase, this means the molecules move faster and therefore collide frequently. The proportion of collision that can overcome the activation energy for the reaction increases with temperature. Phenomenon for sack out of silicon atom fall under microscopic activation energy resulted into new and modified surface morphology. In this view, temperature is a factor for surface ambiguities i.e. roughness. 
AFM measurements have been employed for surface morphology at varying temperatures viz.: $75^{\circ}$ and $95^{\circ}$ C. Fig. 3 summarize these results. Based on the findings smooth surface is found at room environment and rougher surface is evolved during thermal treatment. Although present etchant is relatively fast compared to other etchants, it can produce a poor surface finish as reported in literature (Abott et al., 1993). Thus, Fast etched planes covered with irregularities such as microscopic pyramids (Schwartz and Robbin, 1961).

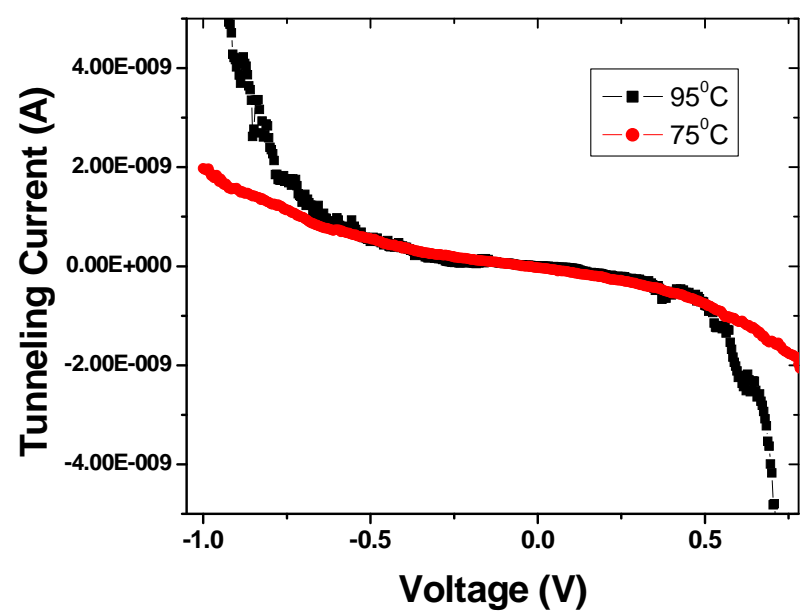

Fig 4: Tunneling spectra of etched silicon at $75^{\circ} \mathrm{C}$ and at $95^{\circ} \mathrm{C}$

The resolution of scanning tunneling microscope (STM) is direct consequence of the quantum mechanical tunneling between the tip and surface. Tunneling current depend both on the width and height of the barrier and on the electronic structure of the tip and surface. Since tip is remains fixed then we know varieties of surface electronic properties of the sample.

Let the distance between tip and sample is ' $d$ ' with work functions for sample and tip is ' $\phi_{s}$ ' and ' $\phi_{t}$ ' respectively and positively bias voltage ' $V$ ' applied to sample. Work function is an extra amount of energy above the fermi energy required to move an electron away from the surface from fermi level $\left(\mathrm{E}_{\mathrm{f}}\right)$ to the vacuum. Quantum mechanical tunneling, however, can occur due to the overlap of electron wave functions that span the gap. The probability for an electron on the tip at energy ' $E$ ' with respect to the ' $E_{f}$ ' to tunnel to the surface can be approximated as, $T \approx \exp \left\{-2 d\left[\frac{2 m}{\hbar^{2}}\left(\bar{\phi} \cdot-E-\frac{e V}{2}\right)\right]^{\frac{1}{2}}\right\}$ (Whithan, 2004).

For small bias voltages, the probability of tunneling for tip electrons near ' $E_{f}$ ' can be further approximated as $T \approx \exp \left\{-2 d\left(\frac{2 m \bar{\phi}}{\hbar^{2}}\right)^{\frac{1}{2}}\right\}$. Where ' $\bar{\phi}$ ' is the average work function 
of the tip and the sample, ' $m$ ' is the electron mass, ' $e$ ' is the electron charge, and ' $\hbar$ ' is plank constant. This means when an electrical voltage ' $V$ ' is applied between sample and tip, net current depend on tip-sample distance ' $d$ ', on voltage ' $V$ ' and height of the barrier ' $\phi$ ' as

$$
I(d) \approx \text { cons } \tan t \times e V \cdot \exp \left\{-2 d\left(\frac{2 m \bar{\phi}}{\hbar^{2}}\right)^{\frac{1}{2}}\right\} \approx \sigma_{\text {tunnel }} V ; \sigma \text { - conductivity tensor }
$$

This equation shows that the tunneling current obeys Ohm's law, i.e., current ' $I$ ' is proportional to the voltage ' $V$ '. It depends exponentially with distance ' $d$ '. Above equation shows that the tunneling current depends strongly on the bias voltage and the distance that it is dominated by the contribution flowing between the last atom of the tip and the nearest atom in the sample.

As we increase the bias voltage ' $V$ ' the potential barrier between tip and sample is going on reducing. The probability that an electron will jump from tip to sample therefore will

be higher. The current amplification factor “ $\exp \left\{2 d\left[\frac{2 m}{\hbar^{2}}\left(\frac{e V}{2}\right)\right]^{\frac{1}{2}}\right\}$ " is suddenly come up

with sharp increase in tunneling current, that's what we experimentally observed. At higher bias voltage ' $V$ ' tunneling current $\exp \left\{2 d\left[\frac{2 m}{\hbar^{2}}\left(E+\frac{e V}{2}\right)\right]^{\frac{1}{2}}\right\}$ follows step function as we found in our experiment.

\section{CONCLUSIONS}

Work identifies thermal agitation is a prime factor which controls ER and activation energy as well as surface morphology of the crystal. ER is slow in (111) face and fast in (110) face. Activation energy is more in (111) and less in (100). Removal of silicon atom should have many practical problems and hence depend on quantum probability laws. Anisotropy of $\mathrm{KOH}$ etching allow us a precious control of lateral dimension of the microstructure bound by (111) planes (Oosterbroek et al., 2000) to form enough foundation for miniaturized devices with high conductance. As we found, band gap is concise with temperature evolution so that conductance is going to be higher. Tunneling spectra, i.e., dependence of tunneling current with bias voltage, one can get information at an atomic level of the sample surface. Since tunneling occurs over an area $<1 \mathrm{~nm}^{2}$ on the sample surface.

\section{ACKNOWLEDGEMENTS}

Thanks for J. Akhtar, Scientist CEERI, Pilani, for preliminary samples. Thanks for Prof. P. Sen, School of Physical Sciences, JNU for performing experiment in his lab. Thanks for University Grants Commission, Kathmandu, for support to the Author. 


\section{REFERENCES}

1. Abott A.P., Campbell S.A, Satherley J. and Schiffrin D.J., 1993. J. Electroanal Chem. 348: 473.

2. Ashcroft N.W. and Mermin N.D., 1988. Sold State Physics, CBS Publishing Asia Ltd, 396.

3. Campbell S.A., Cooper K, Dixon L, Earwaker R, Port S. N. and Schiffrin D. J., 1995, J. Micromech Microeng. 5: 209.

4. Cottam G.M. et al., 1989. Introduction to Surface and Super lattice Excitations, Cambridge University Press, Cambridge NY 12.

5. $\quad$ Finne R.M. and Klein D.L., 1967. J. Electrochem. Soc: Solid State Science, 114: 965.

6. Gosalvez M.A. and Nieminen R.M., 2003. Physical Review E 68: 031604.

7. Hennry Wise and Jacques Oudar, 1990. Material Concepts in Surface Reactivity and Catalysis, Academic Press Inc.

8. James Marchettri, Yie He, Olaf Than, Sandeep Akkaraju, 1998. SPIES's Symposium on Micromachining and Microfabrication, Micromachimed Devices and Components, Sanate Clara, CA, USA.

9. Kenneth E, Bean, 1978. IEEE Transaction on Electron Devices, 25: 1185.

10. Kovacs G T A, 1998. Micromachined Transducers Sourcebook, Boston, M.A., McGraw-Hill.

11. Lee D.B., 1969. Journal of Applied Physics, 40: 4569.

12. Mayer H.P., 1998. Introduction to Solid State Physics, Viva Books Pvt. Ltd, New Delhi 426.

13. Oosterbroek RE, Berenschot J.W., Jansen V. H., Nijdam A.R., Pandraud G., Berg A.V.D. and Elwenspoek M.C., 2000. J. MEMS, 9:390.

14. Perterson K.E., 2001. Silicon as a mechanical material, Proc IEEE 70: 420

15. Roher Gregory S., 2001. Structure and Bonding in Crystalline Materials, Cambridge University Press 410.

16. Schwartz B and Robbin H, 1961. J. Electrochem. Soc., 108:365.

17. Van Vlack, 1970. Material Science for Engineers, Addison-Wesley Publishing Company, Reading, Massachusetts, 68. 
18. Wang C.Z., Pan B. C., and Ho K. M., 1999. J. Phys. Condens. Matter 11: 2043.

19. Wenspoek M. and Jansen H., 1998. Silicon Micromachining, Cambridge University Press 26.

20. Whithan L. J. Tunneling Microscopy and Spectroscopy, Navil Research laboratory, Washington, DC 20375.

21. www.comppub.com/publ/MEM2000/M41.03.pdf. 